\title{
Risk of thrombosis in human atherosclerotic plaques: role of extracellular lipid, macrophage, and smooth muscle cell content
}

\author{
Michael J Davies, Peter D Richardson, Neville Woolf, David R Katz, Jessica Mann
}

\begin{abstract}
Objective-To assess the size of the lipid pool and the number of smooth muscle cells and monocyte/macrophages in human aortic plaques that were intact and to compare the results with those in aortic plaques undergoing ulceration and thrombosis.
\end{abstract}

Design-The lipid pool was measured as a percentage of the total cross sectional area of the plaque. Immunohistochemistry was used to identify cell types (monocytes/macrophages $(M \varphi)$ by EBM11 and HAM56, smooth muscle cells by $\alpha$ actin). The area of the tissue occupied by each cell type was measured by quantitative microscopy in the peripheral (shoulder) area of the plaque and the plaque cap. Absolute counts of each cell type were expressed as the ratio of SMC:M Material-Aortas were obtained at necropsy from men aged less than 69 years who died suddenly (within 6 hours of the onset of symptoms) of ischaemic heart disease. 155 plaques from 13 aortas were studied. Four aortas showed intact plaques only (group $A, n=31$ ). Nine aortas showed both intact plaques (group B, $n=79$ ) and plaques that were undergoing thrombosis (group $C, n=45$ ).

Results-In $41(91 \cdot 1 \%)$ of the 45 plaques undergoing thrombosis (group C) lipid pools occupied more than $40 \%$ of the cross sectional area of the plaque. Only $12(10.9 \%)$ of the 110 intact plaques (groups A + B) had lipid pools of this size. The mean size of the lipid pool in plaques of groups $A, B$, and $C$ was $12 \cdot 7 \%$, $27 \cdot 3 \%$ and $56.7 \%$ respectively. Compared with intact plaques those undergoing thrombosis contained a smaller volume of smooth muscle cells $(2.8 \% \quad v \quad 11.8 \%)$ and a larger volume of monocytel macrophages $(13.7 \% \quad v \quad 2.9 \%)$ in the plaque cap. The ratio of the number of smooth muscle cells to monocytes/ macrophages was 7.8 in group A plaques, $4 \cdot 1$ in group $B$ plaques, and 1.0 in group $C$ plaques. This gradient was the result of an absolute increase in monocytel macrophages and an absolute decrease in smooth muscle cells.

Conclusions-In the aorta ulceration and thrombosis were characteristic of plaques with a high proportion of their volume occupied by extracellular lipid, and in which there was a shift toward a preponderance of monocyte/macrophages compared with smooth muscle cells in the cap.

(Br Heart f 1993;69:377-381)

The human atherosclerotic plaque has come under intense study to clarify why it is complicated by the sudden episodes of thrombosis which lead directly to considerable morbidity. ${ }^{12}$ The importance of plaque fissuring, or cracking, or ulceration has become increasingly recognised in pathological material, ${ }^{3-5}$ in both in vivo and in postmortem angiographic studies ${ }^{67}$ and recently at angioscopy. ${ }^{89}$ Pathological studies have emphasised the importance of a central lipid pool in the process of plaque fissuring ${ }^{1011}$ and this has been confirmed by in vivo intravascular ultrasound. ${ }^{12}$

These studies do not, however, indicate whether there is a critical size of the lipid pool at, or above which, the risk of fissuring is significantly increased. Nor do they provide information on the type and number of cells which make up the plaque.

Plaques that fissure are of the advanced or raised fibrolipid type ${ }^{13}$ and have a characteristic microanatomy. The core of the plaque consists of extracellular lipid separated from the lumen by a fibrous tissue cap. Within this cap there are smooth muscle cells and monocyte/macrophages. The margins of the lipid core also contain monocytes/macrophages, concentrated at the lateral or shoulder area of the plaque. This concentration has led to the suggestion that plaques grow centrifugally and are most active at their edges. ${ }^{14}$

We describe the major cellular constituents of different parts of advanced aortic plaques in terms of their quantitative relation to lipid cores of different sizes and whether the plaque had undergone thrombosis.

\section{Methods}

At necropsy we studied aortas from men aged less than 69 who died suddenly and who had 

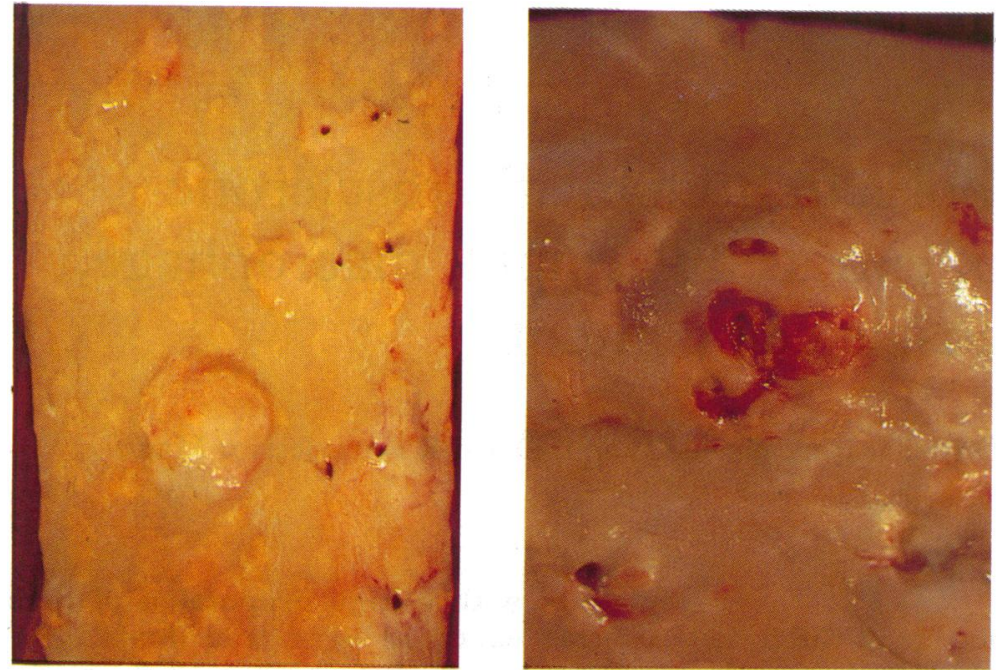

Figure 1 Contrast between an intact advanced aortic plaque (left), in which the surface is opaque and smooth, and early ulceration (right), in which thrombus has formed over the plaques. A large part of the plaque, however, is not covered by thrombus.

significant stenosis caused by coronary atherosclerosis without any other apparent cause of death. The aorta was slit open longitudinally and, the intima was washed with physiological saline and then examined by the naked eye. All the advanced fibrolipid atherosclerotic plaques that were raised above the surface and had a long axis of $>0.75 \mathrm{~cm}$ in the thoracic and abdominal aorta from the left subclavian to the renal arteries were studied. Flat lesions (fatty streaks) were not studied. Plaques were classified as those with a smooth, apparently intact intimal surface and those with an area of thrombosis and focal ulceration at one end that left most of the plaque intact (fig 1). We did not study plaques that were extensively ulcerated, covered by thrombus and in which there was no

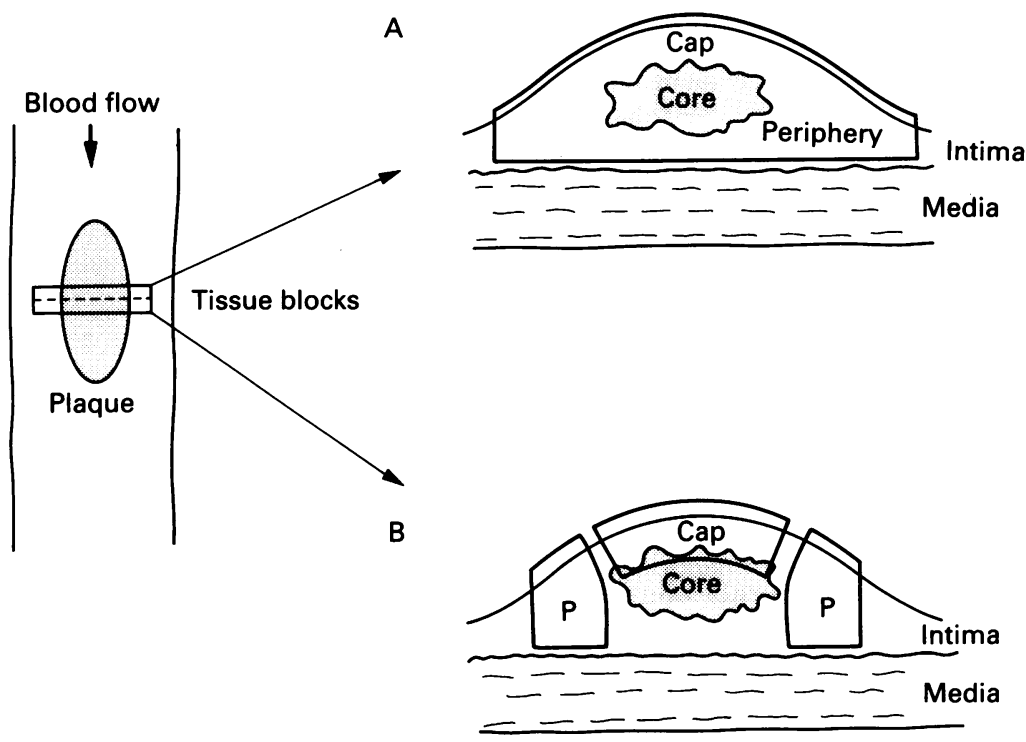

Figure 2 Method of plaque analysis. $A$ tissue block was taken across the short axis of the plaque. Proximate histological sections were taken and stained for lipid, collagen, and the different cell types. The sections were examined in a quantifying microscope. $(A)$ The outline of the plaque (red line) was defined by the user. Within this red line the area occupied by the lipid core was measured under very low power microscopy and expressed as a percentage of the whole area. (B) User defined fields (outlined in red) encompassing the cap tissue and peripheral portions of the plaque. Within the peripheral and cap fields we measured the area occupied by macrophage and smooth muscle cytoplasma and counted their numbers. residual intact plaque structure. Two adjacent transverse $2 \mathrm{~mm}$ thick blocks of tissue were taken through the centre of the plaque avoiding areas of ulceration (fig 2). One sample was frozen in liquid nitrogen and $6 \mu \mathrm{m}$ thick sections were cut on a cryostat. The other sample was fixed in formaldehyde and processed to paraffin wax.

Histological sections from the paraffinembedded material were stained for collagen by the Sirius Red method and by the haematoxylin and eosin method. In frozen material lipid was stained by the Sudan III method. Monocytes/macrophages were identified immunohistochemically with antibody EBM1 $1^{15}$ and antibody HAM56. Smooth muscle cells were identified by antibodies to smooth muscle $a$ actin. ${ }^{16}$ All these antibodies mark the cytoplasm of the cells and allow the area of the section that is stained positively to be used as a measure of the volume of the tissue occupied by different cell types. The immunohistochemical reaction was visualised by the APAAP method. ${ }^{17}$ Tissue sections were quantified by an AMS quantifying microscope and an Optomex programme run on an IBM 55-SX computer. The outline of each plaque, excluding the media, was drawn manually from the haematoxylin and eosin stained section and displayed on the TV monitor screen (fig 2). Within this overall plaque outline we drew in fields, red lines on (fig 2), delineating the peripheral (shoulder) area adjacent to more normal intima and the plaque cap. All these outlines were then retained on the monitor screen. Images of immediately adjacent histological sections stained for collagen, lipid, smooth muscle, and monocyte/macrophages were then successively registered on these outlines to build up a picture of the constituents of the plaque. The lipid pool area was defined as the proportion of the total plaque cross sectional area occupied by extracellular lipid alone (fig 2). For each delineated field (shoulder/periphery or cap) within the plaque the areas occupied by cell cytoplasm of smooth muscle cells and monocyte/macrophages was measured as percentages of the field area. Within the defined fields we counted the total number of nuclei, and the ratio which fell within cytoplasm marking for smooth muscle cells or monocytes/macrophages was also counted. Cell counts were expressed as the number per square millimetre of tissue. Unidentified cells made up less than $10 \%$ in all the samples and this proportion was similar in all the plaque groups.

In all, we analysed 155 plaques from 13 aortas. Four of the aortas contained intact plaques only and there was no thrombus at any site in the aorta; in the other nine aortas there was a mixed population of intact and ulcerated plaques. Thirty one plaques were intact and came from the four aortas in which there were no thrombotic plaques (group A). There were 79 intact plaques (group B) and 45 ulcerated plaques (group C) which came from the nine aortas in which there were both types of plaque. 
The results are expressed as mean (SEM). We used a two tailed Student's $t$ test (Statsview 512) and a three dimensional plot (DANCAD 3D) programme to compare the areas occupied by smooth muscle, macrophages, and extracellular lipid core in the three different plaque types.

\section{Results}

The area occupied by the lipid core was largest in the group $\mathrm{C}$ plaques and smallest in the group $A$ plaques. The increase in the mean core size from $A$ to $B$ to $C$ was statistically significant (table). Figure 3 shows the differences as plots of individual data points (fig 3). Forty one $(91 \cdot 1 \%)$ of the 45 ulcerated plaques in group $\mathrm{C}$ had lipid cores occupying $>40 \%$ of the cross sectional area of the plaque. Of the intact plaques in group $\mathrm{A}$ and B only $12(10.9 \%)$ of 110 had bigger pools.

Figure 3 Individual data point plots of the percentage lipid content of the three plaque groups. Though there was considerable overlap between the groups the mean values were very different. Only one plaque in group $A$ had a value over $40 \%$ while 41 of the 45 plaques in group $C$ exceeded a value of $40 \%$.

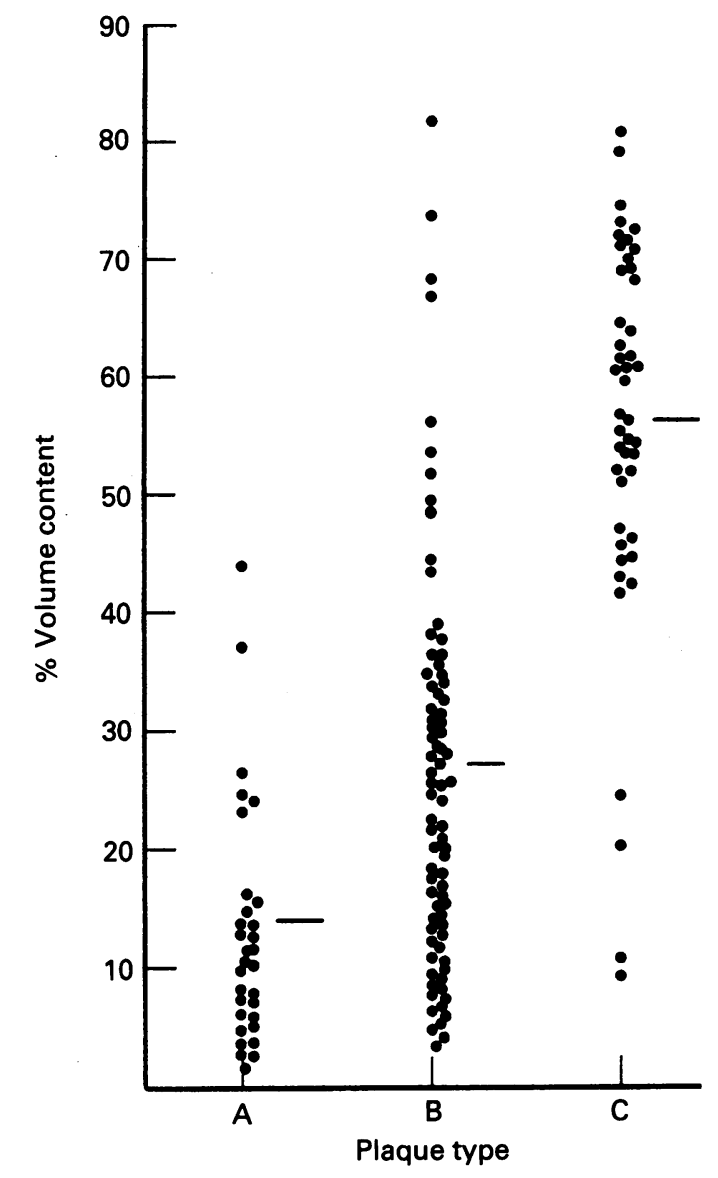

These data reinforce the view that the size of the lipid pool is positively correlated with the risk of ulceration and thrombosis, and that the crucial threshold is $40 \%$ of the plaque cross sectional area in its mid point. Within the intact plaques of group B 11 (13.9\%) of the 79 plaques had lipid pools that exceeded this value. These may be plaques in which ulceration would have occurred in the future.

Within cap tissue the area occupied by monocytes was greatest in plaques of group $C$ but the areas in groups A and B were not significantly different. The area occupied by smooth muscle cells in cap tissue decreased from $A$ to $B$ to $C$. The ratio of absolute numbers of smooth muscle cells to macrophages per unit area was highest in cap tissue of plaques from group $\mathrm{A}$ and lowest in group $\mathrm{C}$. The changes in both occupied area and number density of cell types in the shoulder/periphery region of the plaque paralled those in the cap in a similar direction but were not as big. Figure 4 shows the three dimensional plots linking changes in the lipid core size to changes in the cell populations at both sites in the plaques.

\section{Discussion}

This study relates the lipid core size and smooth muscle and macrophage content of three groups of plaques. Plaques in group A can be regarded as coming from aortas in which there was no tendency to plaque thrombosis while in group $\mathrm{C}$ the plaques were undergoing thrombosis.

The most striking difference between the plaques of group $A$ and $C$ was the size of the central pool of extracellular lipid which exceeded $40 \%$ of the cross sectional area of the plaques in $91.1 \%$ of group C plaques but in only $3 \cdot 2 \%$ of group $A$ plaques. Thus the size of the lipid pool is an important determinant of thrombosis on aortic plaques. It can be questioned whether aortic plaque thrombosis is a paradigm of coronary thrombosis. There are, however, abundant observational, but not quantified data, showing a similar relation between a coronary lipid rich plaques and thrombosis. ${ }^{910}$

The data also show the relation between the plaque type and the cell content at different sites within the plaque. For the cap tissue three dimensional plots show that as the size

Analysis of different types of plaques for lipid pool size and cell density

\begin{tabular}{|c|c|c|c|c|c|}
\hline & Group $A$ & $\begin{array}{l}\text { Significance } \\
A-B\end{array}$ & Group B & $\begin{array}{l}\text { Significance } \\
B-C\end{array}$ & Group C \\
\hline $\begin{array}{l}\text { Lipid core (as \% plaque area) } \\
\text { Area (\%): }\end{array}$ & $12 \cdot 7(1 \cdot 8)$ & $<0.0002$ & $27 \cdot 3(2 \cdot 1)$ & $<0.0001$ & $56 \cdot 7(2 \cdot 6)$ \\
\hline $\begin{array}{l}\text { M } \varphi \text { cap } \\
\text { SMC cap } \\
\text { M } \varphi \text { periphery } \\
\text { SMC periphery }\end{array}$ & $\begin{array}{r}2.5(0.8) \\
16.3(1.9) \\
4.9(0 \cdot 7) \\
13 \cdot 5(1 \cdot 6)\end{array}$ & $\begin{array}{l}0.3343 \text { (NS) } \\
0.4681 \text { (NS) } \\
0.7211 \text { (NS) } \\
0.434 \text { (NS) }\end{array}$ & $\begin{array}{r}2.9(0.4) \\
11.8(1.0) \\
4.9(0.6) \\
11.8(0.9)\end{array}$ & $\begin{array}{l}<0.0001 \\
<0.0001 \\
<0.006 \\
<0.003\end{array}$ & $\begin{array}{r}13 \cdot 7(1 \cdot 6) \\
2 \cdot 8(0 \cdot 6) \\
8 \cdot 9(1 \cdot 2) \\
5 \cdot 8(0 \cdot 7)\end{array}$ \\
\hline $\begin{array}{l}\left.\text { Number (per } \mathrm{mm}^{2}\right): \\
\text { M } \varphi \text { in cap } \\
\text { SMC in cap } \\
\text { M } \varphi \text { in periphery } \\
\text { SMC in periphery }\end{array}$ & $\begin{array}{r}60(17 \cdot 3) \\
183(18 \cdot 3) \\
96(14 \cdot 0) \\
183(19 \cdot 4)\end{array}$ & $\begin{array}{l}0.2878 \text { (NS) } \\
0.8385 \text { (NS) } \\
0.6743 \text { (NS) } \\
0.4938 \text { (NS) }\end{array}$ & $\begin{array}{l}79(7 \cdot 2) \\
186(13 \cdot 5) \\
108(9 \cdot 1) \\
212(14 \cdot 8)\end{array}$ & $\begin{array}{l}<0.0006 \\
<0.0001 \\
<0.002 \\
<0.003\end{array}$ & $\begin{array}{r}142(13 \cdot 9) \\
60(9 \cdot 8) \\
153(14 \cdot 6) \\
113(10 \cdot 9)\end{array}$ \\
\hline $\begin{array}{l}\text { Ratio number } S M C / M \varphi \\
\text { in cap tissue }\end{array}$ & $7 \cdot 8$ & & $4 \cdot 1$ & & $1 \cdot 0$ \\
\hline
\end{tabular}

Entries are mean (standard error of mean). 
Figure 4 Three dimensional plots relating percentage lipid and smooth muscle and macrophage content in the three plaque types within cap tissue and the shoulder region. Every plaque in each group was plotted. The striking feature is that intact plaques in group $A$ had a low lipid content (at the front of the graph) with a high proportion of smooth muscle. In contrast, unstable ulcerated plaques in group $C$ had high lipid (at the back of the graph) and macrophage contents with a striking reduction in smooth muscle content.

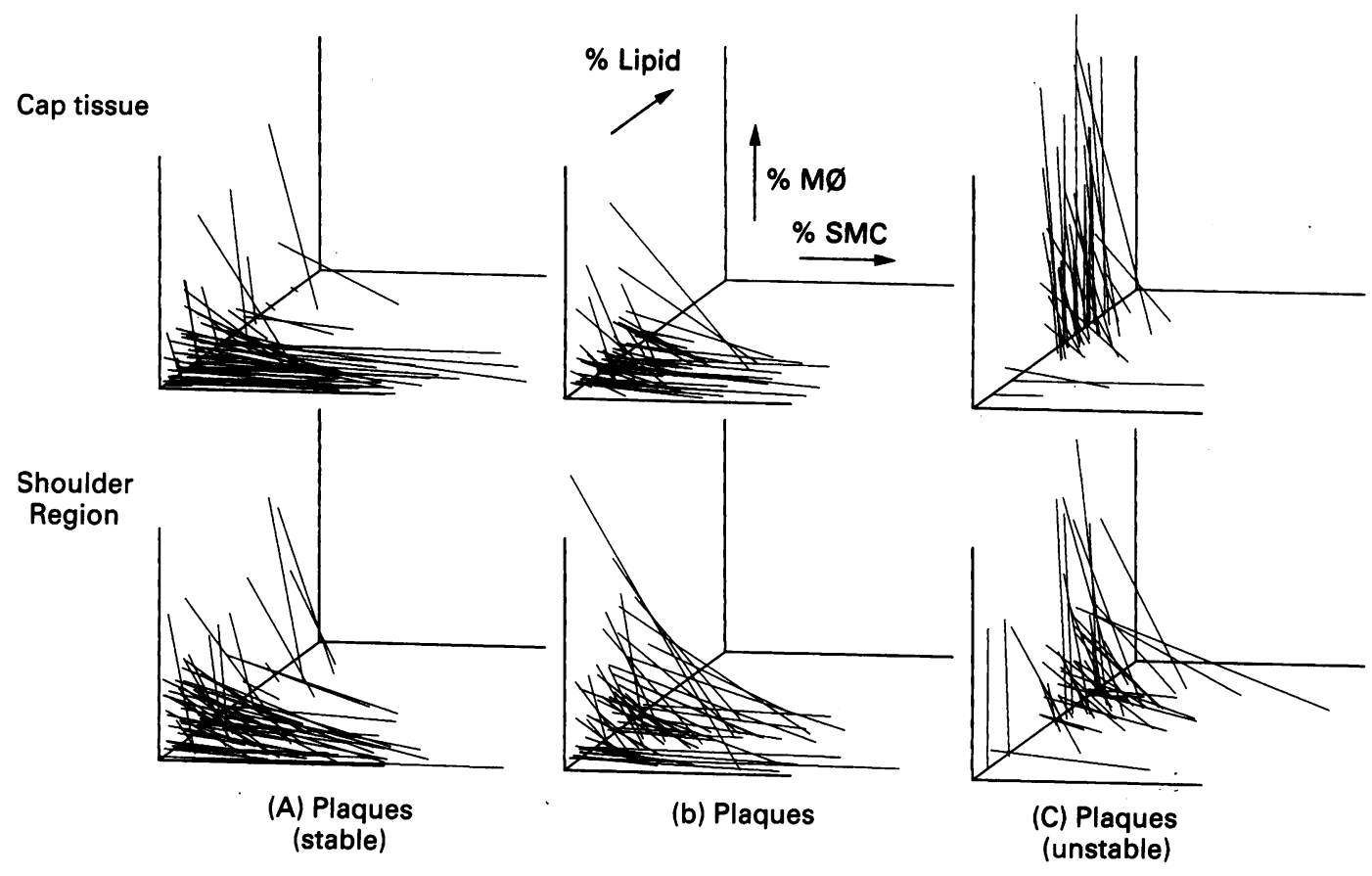

of the lipid pool increased $(0 \%$ is in the front of fig 4) the volume fraction occupied by smooth muscle cells falls, and that of macrophages rises. The total volume fraction occupied by smooth muscle cell and macrophage cytoplasm is $10-25 \%$, the remainder of the tissue being connective tissue matrix. Changes in the shoulder region are virtually identical.

In the three dimensional graphs every plaque is plotted as a line. There is very close concordance between all the plaques in a particular group and no suggestion that the plots are distorted by a particular population of plaques perhaps coming from one or two individuals whose plaques are in some way different.

The sequence of events leading to the quantitative changes observed is not known. It is generally accepted that most foam cells are derived from monocytes that have initially adhered to the intimal aspect of the plaque, which they have then entered. ${ }^{14} 18$ Whether macrophages move laterally within the plaque is not known, though an increased density of macrophages in the peripheral shoulder regions of plaques has been noted previously. ${ }^{19} 20$ Some of the increase in the area fraction of macrophages at higher lipid fractions is associated with an increase in the cell size as macrophages become more distended with lipid, but this is not the whole explanation because there are similar changes in absolute cell number density. Smooth muscle cells contained some lipid but retained their elongated shape.

As in all other tissues, the relative cell populations within plaques may change after selective cell death, by an increase or decrease in the rate of division of one cell type, or by migration into or away from the area. Both monocytes and smooth muscle cells can divide within the plaque ${ }^{21}$ and smooth muscle migration is well recognised, but which of the possible mechanisms brings about the shifts in cell population within cap tissue is unknown.

More particularly, it is not known whether shifts in cell populations precede or follow upon the alteration in lipid content of the plaque, or even whether each is driven by independent forces. The men we studied had not been taking lipid-reducing pharmaceutical agents, so it is unlikely that the results include cases in which the lipid content of the plaque had increased beyond $40 \%$ and had subsequently been reduced.

The changes described in the plaques may largely explain the propensity to ulceration and thrombosis. Previous studies of the mechanical properties of aortic plaque cap tissue in vitro showed an association between the number of macrophages containing lipid and cap fracture..$^{22}$ The mechanical strength of the plaque cap tissue depends on the amount, and organisation, of the connective tissue matrix proteins-including collagen, elastin, and proteoglycans. These proteins in turn depend on metabolism and synthesis by smooth muscle cells. A loss of smooth muscle cells would in time lead to degeneration and fragmentation of the connective tissue, but a more active dissolution by proteolytic enzymes released by both smooth muscle cells and macrophages is also possible. ${ }^{23}$ The association of smooth muscle proliferation with post-angioplasty stenosis has led to the view that it is an undesirable aspect of atherosclerosis. Our study suggests that smooth muscle proliferation is an integral part of plaque stability and repair and is thus necessary to some extent.

Ulceration and thrombosis of aortic plaques can be regarded a paradigm of plaque fissuring in the coronary arteries but there are some important differences. Ulcerated and thrombotic plaques seem to persist and become chronic in the aorta and carotid 
arteries. ${ }^{24}$ Chronic ulceration and thrombosis are far less common in the coronary arteries. In our study we eliminated aortic plaques that had become an ulcer crater filled with thrombus and analysed only plaques that were largely still intact. It could be argued that the changes we have quantified are merely secondary to the process of ulceration. Against this view is the fact that group B plaques, which were still intact, had some characteristics that lay between those of groups $A$ and C.

The changes that we have described indicate that three processes-lipid accumulation, both extracellular and associated with an increase in lipid laden macrophages, and disturbances of the reparative smooth muscle proliferation in the cap-predispose to fissuring. Animal studies of regression suggest all these processes may be reversed by plasma lipid lowering. ${ }^{25-27}$ If the same were true in humans lipid lowering might improve plaque stability with a consequent reduction in the acute sequelae of plaque fissuring including unstable angina and acute myocardial infarction in the coronary circulation and embolic stroke from ulcerated carotid plaques. Data are beginning to accumulate that plasma lipid lowering does lead to a reduction in the number of subsequent acute ischaemic events in men. ${ }^{28}$ The present study is consistent with the view that this effect is mediated by either the prevention of the formation new lipid rich plaques or the restoration of smooth muscle proliferation within existing plaques, which makes them more stable.

1 Fuster V, Badimon L, Badimon J, Chesebro J. Mechanisms of Disease: The pathogenesis of coronary artery disease and the acute coronary syndromes I. artery disease and the acute
$N$ Engl $₹$ Med 1992;326:242-50.

2 Fuster V, Badimon L, Badimon J, Chesebro J. The pathogenesis of coronary artery disease and the acute coronary syndrome II. N Engl $f$ Med 1992;326:310-8.

3 Constantinides P. Plaque fissures in human coronary thrombosis. F Atheroscl Res 1966;6:1-17.

4 Davies MJ, Thomas AC. Plaque fissuring-the cause of acute myocardial infarction, sudden ischaemic death, and crescendo angina. $\mathrm{Br}$ Heart $\mathcal{f}$ 1985;53:363-73.

5 Falk E. Unstable angina with fatal outcome: dynamic coronary thrombosis leading to infarction and/or sudden death. Circulation 1985;71:699-708.

6 Levin D, Fallon J. Significance of the angiographic morphology of localized coronary stenosis: histopathologic correlations. Circulation 1982;66:316-20.

7 Ambrose J, Winters S, Arora R, et al. Coronary angiograph morphology in acute myocardial infarction: link between the pathogenesis of unstable angina and myocardial infarction. $\mathcal{F} \mathrm{Am}$ Coll Cardial 1985;6: 1233-8.

8 Forrester J, Litvak F, Grundfest W, Hickey A. A perspective of coronary disease seen through the arteries of a living man. Circulation 1987;75:505-13.

9 Mizuno KM Satomura K, Miyamoto A, Arakawa K, Shibuya T, Arai T, Kurita A, Nakamura H, Ambrose JA. Angioscopic evaluation of coronary-artery thrombi in acute coronary syndromes. $N$ Engl $f$ Med 1992; 326:287-91.

10 Tracy R, Devaney K, Kissling G. Characteristics of the plaque under a coronary thrombus. Virchows Arch $[A]$ 1985;405:411-27.

11 Richardson $\mathrm{P}$, Davies $\mathrm{M}$, Born $\mathrm{G}$. Influence of plaque configuration and stress distribution on fissuring of configuration and stress distribution on fissuring of

12 Nissen S, Gurley J, Booth D, et al. Differences in ultravascular plaque morphology in stable and unstable patients cular plaque morphology in stab

13 Stary $\mathrm{H}$. Evolution and progression of atherosclerotic lesions in coronary arteries of children and young adults. Arteriosclerosis 1989;9:1-19.

14 Aqel N, Ball R, Waldmann H, Mitchinson M. Monocytic origin of foam cells in human atherosclerotic plaques. Atherosclerosis 1984;53:265-71.

15 Kelly P, Bliss E, Morton J, Burns J, McGee J. Monoclonal antibody EBM11. High cellular specificity for human macrophages. $\mathcal{F}$ Clin Pathol 1988;41:510-5.

16 Skalli O, Ropraz P, Trzeciak A, Benzonana G, Gillessen D, Gabbiani G. A monoclonal antibody against alphasmooth muscle actin: a new probe for smooth muscle differentiation. $f$ Cell Biol 1986;103:2787-96.

17 Cordell J, Falini B, Erber W. Immunoenzymatic labelling of monoclonal antibodies using immune complexes of alkaline phosphatase and monoclonal antialkaline phosphatase (APAAP complexes). $\mathcal{F}$ Histochem Cytochem 1984;32:219-29.

18 Faggiotto A, Ross R. Studies of hypercholesterolaemia in non-human primates. II Fatty streak conversion to fibrous plaque. Arteriosclerosis 1984;4:341-56.

19 Jonasson L, Holm J, Skalli O, Bondjers G, Hansson K. Regional accumulations of $\mathrm{T}$ cells, macrophages and smooth muscle cells in the human atherosclerotic plaque. Arteriosclerosis 1986;6:131-8.

20 Gown A, Tsukakda T, Ross R. Human atherosclerosis II immunocytochemical analysis of the cellular composition of human atherosclerotic lesions. Am $\mathfrak{f}$ Pathol 1986;125:191-207.

21 Wilcox J. Analysis of local gene expression in human atherosclerotic plaques by in-situ hybridisation. Trends Cardiovasc Med 1991;1:17-24.

22 Lendon C, Davies $M$, Born G, Richardson $P$. Atherosclerotic plaque caps are locally weakened when macrophage density is increased. Atherosclerosis 1991;65: 302-10.

23 Henney A, Wakeley P, Davies $M$, et al. Localization of stromelysin gene expression in atherosclerotic plaques by in situ hybridization. Proc Natl Acad Sci USA 1991; 88:8154-8.

24 Amarenco P, Duyckaerts C, Tzourio C, et al. The prevalence of ulcerated plaques in the aortic arch in patients with stroke. $N$ Engl f Med 1992;326:221-5.

25 Small DM, Bond MG, Waugh D, Prack M, Sawyer JK. Physiochemical and histological changes in the arterial wall of the non-human primates during progression and wall of the non-human primates during progression and
regression of atherosclerosis. $f$ Clin Invest 1984;73: regression $1590-1605$.

26 Stary $H$. Progression and regression of experimental atherosclerosis in rhesus monkeys. In: Goldsmith E, Morr-Hankowsky J, ed. Medical primatology. Basel: S Karger, 1972:356-7.

27 Armstrong ML, Heistad DD, Megan MB, Lopez JA. Reversibility of atherosclerosis. Cardiovasc Clin 1990;20: 113-26.

28 Brown G, Albers J, Fisher L. Regression of coronary artery disease as a result of intensive lipid-lowering therapy in men with high levels of apolipoprotein B. $N$ Engl $¥$ Med 1990;323:1289-98. 